\title{
Ultrasound and necropsy study of periventricular haemorrhage in preterm infants
}

\author{
W SZYMONOWICZ, K SCHAFLER, L J CUSSEN, AND V Y H YU \\ Departments of Paediatrics and Pathology, Queen Victoria Medical Centre, Melbourne, Australia
}

SUMmary The diagnostic accuracy of cerebral ultrasound for periventricular haemorrhage was determined by comparing this with necropsy findings in 30 preterm neonates of 30 weeks' gestation or less and birthweight under $1500 \mathrm{~g}$. Ultrasound gave an accurate diagnosis of $85 \%$ in infants with germinal layer haemorrhage, $92 \%$ in intraventricular haemorrhage, and $97 \%$ in intracerebral haemorrhage. False positive errors were caused by vascular congestion; false negative errors occurred when the maximum dimension of haemorrhage was less than $3 \mathrm{~mm}$. Cerebral ultrasound gave a diagnostic accuracy of $63 \%$ for periventricular leucomalacia. False negative errors occurred when periventricular leucomalacia was microscopic or when it was out of range of the scanner.

The maximum width of the germinal layer was measured in 77 neonates of gestational age 23 to 36 weeks who died and had no periventricular haemorrhage at necropsy. The progressive involution of the germinal layer with increasing gestational age paralleled the steady decrease in incidence of periventricular haemorrhage diagnosed over the same gestational age range. Neonates of the youngest gestational age who had the most extensive germinal layers also had the highest risk for periventricular haemorrhage.

The extremely preterm neonate is at high risk of periventricular haemorrhage. ${ }^{\prime}$ Comparison of cerebral ultrasound diagnoses with necropsy findings have been reported in very few instances ${ }^{2+4}$ and sources of error have been described in only one study. ${ }^{2}$ Although many perinatal factors have been identified as antecedents of periventricular haemorrhage, ${ }^{56}$ the germinal layer is the most common site of origin of bleeding. ${ }^{7}$ In the present study we have investigated the diagnostic accuracy of cerebral ultrasound for periventricular haemorrhage and periventricular leucomalacia by comparing ultrasound and necropsy findings. In addition we have documented the natural involution of the germinal layer with increasing gestational age in preterm neonates and have compared this with the incidence of periventricular haemorrhage diagnosed by cerebral ultrasound.

\section{Patients and methods}

Thirty preterm neonates of less than 30 weeks" gestation whose birthweight was under $1500 \mathrm{~g}$ were studied to determine the diagnostic accuracy of cerebral ultrasound for periventricular haemor- rhage. All infants were scanned within two hours of death or shortly after death and all underwent necropsy examination of the brain. Cerebral ultrasound scans were performed by one of us (WS) using a real time, linear array scanner (Toshiba SAL $1205-\mathrm{MHz}$ probe) through the anterior fontanelle in coronal and sagittal planes and through the skull bones in axial and coronal planes. The imaging diagnosis was made and the size of the haemorrhage or cyst estimated independently (WS), without knowledge of necropsy findings.

At necropsy the brain was removed and suspended in $10 \%$ formalin for at least two weeks before sectioning. An external assessment of maturity and inspection for subarachnoid haemorrhage was done on each occasion (KS, LC). The cerebral hemispheres were cut coronally at approximately $1 \mathrm{~cm}$ intervals. The brain stem and cerebellum were sectioned separately. Tissue sections were stained with hematoxylin eosin. Germinal layer haemorrhage was defined as either macroscopic or microscopic bleeding into the germinal layer over the caudate nucleus. Intraventricular haemorrhage was defined as free blood or blood clot in the lateral ventricles or hemosiderin staining of the ventricular 
walls. Intracerebral haemorrhage was defined as bleeding into the brain parenchyma. The presence of periventricular leucomalacia, whether or not accompanied by cystic degeneration, was also noted. Diagnosis of periventricular haemorrhage and periventricular leucomalacia at necropsy (KS, LC) was made without prior knowledge of the imaging results.

Ultrasound and necropsy comparisons for the right and left sides of the brain were analysed separately, thereby giving 60 sites of possible haemorrhage. Germinal layer, intraventricular, and intracerebral haemorrhages were considered as separate entities. Agreement was found when the imaging diagnosis correlated with the necropsy findings in respect of site and size. A false negative error occurred when no haemorrhage was seen on scanning but was present at necropsy. A false positive error occurred when the haemorrhage diagnosed on scanning was not found at necropsy. The same analysis was done for periventricular leucomalacia.

In addition, the brains of 77 preterm infants of gestational age 23 to 36 weeks, who died in the neonatal period and in whom periventricular haemorrhage was not found at necropsy, were studied microscopically to determine the size of the germinal layer. Using a Leitz eyepiece $10 \times 10$ grating calibrated with a Leitz micrometer, the maximum width of the germinal layer in coronal sections taken at the level of the body of the lateral ventricle was measured in these neonates. The mean and standard deviation (SD) of the width of the germinal layer for each gestational group was calculated.

\section{Results}

The birthweight of the neonates in the ultrasound and necropsy comparison study was mean (SD) $827 \mathrm{~g}$ $(289 \mathrm{~g})$ and their gestational age was mean (SD) 26 weeks ( 2 weeks). There were twice as many boys as girls. The median age at death was 4 days (range, 15 minutes to 165 days). Often there was more than one cause of death. Other than periventricular haemorrhage, necropsy findings included hyaline membrane disease $(43 \%)$, infection $(23 \%)$, asphyxia $(13 \%)$, pulmonary haemorrhage $(13 \%)$, necrotizing enterocolitis $(13 \%)$, bronchopulmonary dysplasia $(7 \%)$, and congenital lung or heart malformations $(7 \%)$. Clinical signs of intracranial bleeding (seizures, apnoea, reduction in haemoglobin concentration greater than $3 \mathrm{~g} / \mathrm{dl}$ ) occurred in 7 of the 21 neonates $(33 \%)$ who had a necropsy diagnosis of periventricular haemorrhage. Two thirds of the neonates with periventricular haemorrhage were therefore asymptomatic.
Neuropathological examination of the brains of the 30 neonates showed no haemorrhage in 10 $(33 \%)$, germinal layer haemorrhage in $18(60 \%)$, intraventricular haemorrhage in $17(57 \%)$, intracerebral haemorrhage in $11(33 \%)$, subarachnoid haemorrhage in $16(53 \%)$, and cerebellar haemorrhage in $6(20 \%)$. Germinal layer haemorrhage was bilateral in 13 neonates, unilateral in five, and ranged in size from microscopic to $9 \mathrm{~mm}$ in width. Intraventricular haemorrhage was bilateral in 11 neonates, unilateral in 6 , and was associated with ventriculomegaly in $7(41 \%)$. Intracerebral haemorrhage was unilateral in 9 neonates and bilateral in two; it occurred in the frontoparietal region in 9 infants and in the temporo-occipital region in four. Evidence of periventricular leucomalacia was present in 8 neonates $(27 \%)$, five of whom also showed cystic degeneration.

Good agreement between ultrasound imaging and necropsy findings occurred in $91 \%$ overall. Results of the correlation for germinal layer, intraventricular, and intracranial haemorrhage independently are shown in Table 1. The diagnostic accuracy for germinal layer haemorrhage was $85 \%$. False negative errors occurred in four instances (two lesions were microscopic and two lesions measured 2 $\mathrm{mm} \times 1 \mathrm{~mm}$ ) because the scanner's resolution limit was $3 \mathrm{~mm}$. False positive errors in five instances were due to vascular congestion over the germinal layer visible macroscopically at necropsy in three infants (Fig. 1) and due to distortion produced by a very large intraventricular haemorrhage in the other two. The diagnostic accuracy of cerebral ultrasound for intraventricular haemorrhage was $92 \%$ (Fig. 2). The five false positive errors were due to vascular congestion of the choroid plexus, visible macrosco-

Table 1 Correlation of necropsyproved lesions with ultrasound

\begin{tabular}{|c|c|c|}
\hline & No & $\%$ \\
\hline Total sites & 60 & 100 \\
\hline \multicolumn{3}{|c|}{ Germinal laver haemorrhage } \\
\hline Correct yes & 271 & \\
\hline Correct no & 24 & 85 \\
\hline False negative & 4 & 7 \\
\hline False positive & 5 & 8 \\
\hline \multicolumn{3}{|c|}{ Intraventricular hacemorrhage } \\
\hline Correct yes & 281 & \\
\hline Correct no & 271 & 92 \\
\hline False negative & 0 & () \\
\hline False positive & 5 & 8 \\
\hline \multicolumn{3}{|c|}{ Intracranial haemorrhage } \\
\hline Correct yes & 111 & 47 \\
\hline Correct no & 471 & 97 \\
\hline False negative & 2 & 3 \\
\hline False positive & 0) & 0 \\
\hline
\end{tabular}



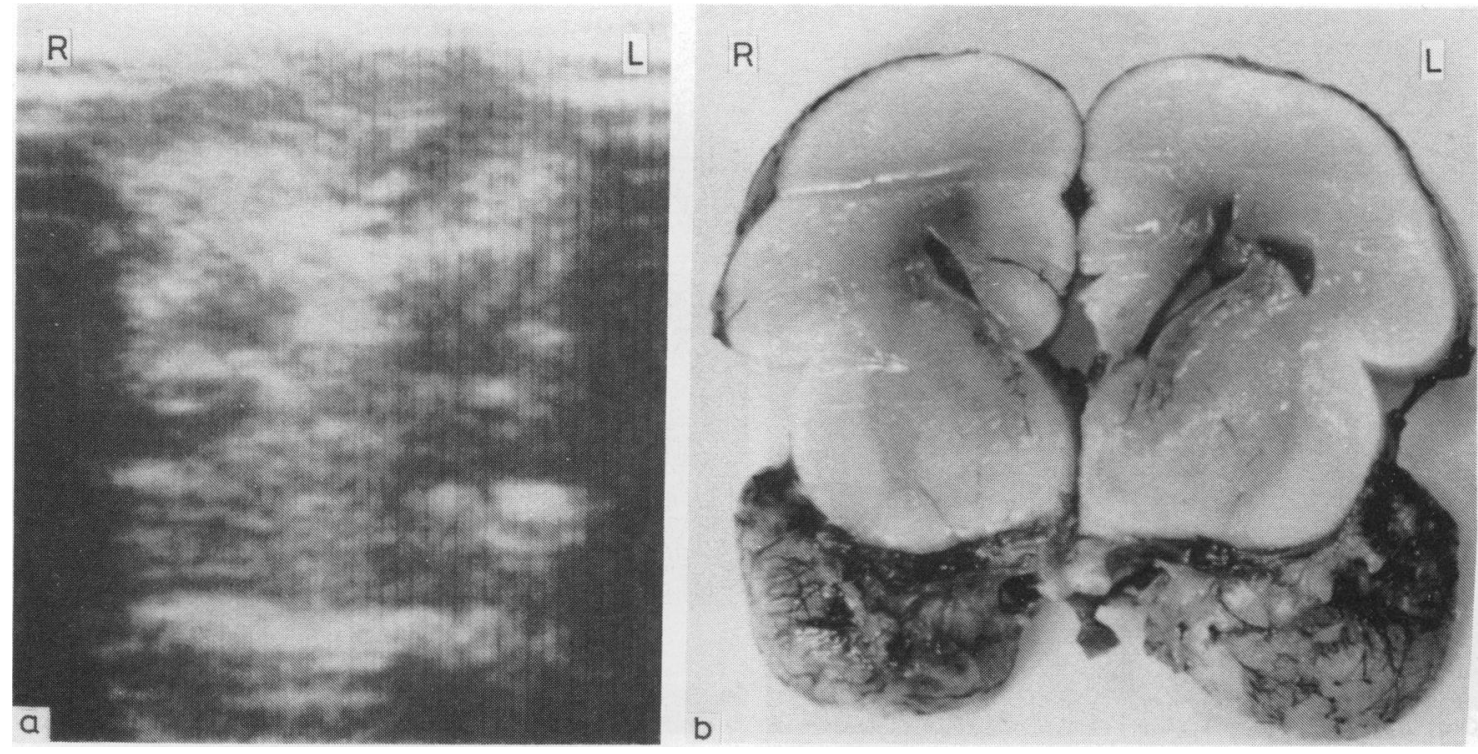

Fig. 1 False positive error (a)-abilateral germinallaverhaemorrhage was diagnosed on ultrasound; (b)-this was found to be due to vascular congestion at necropsy.

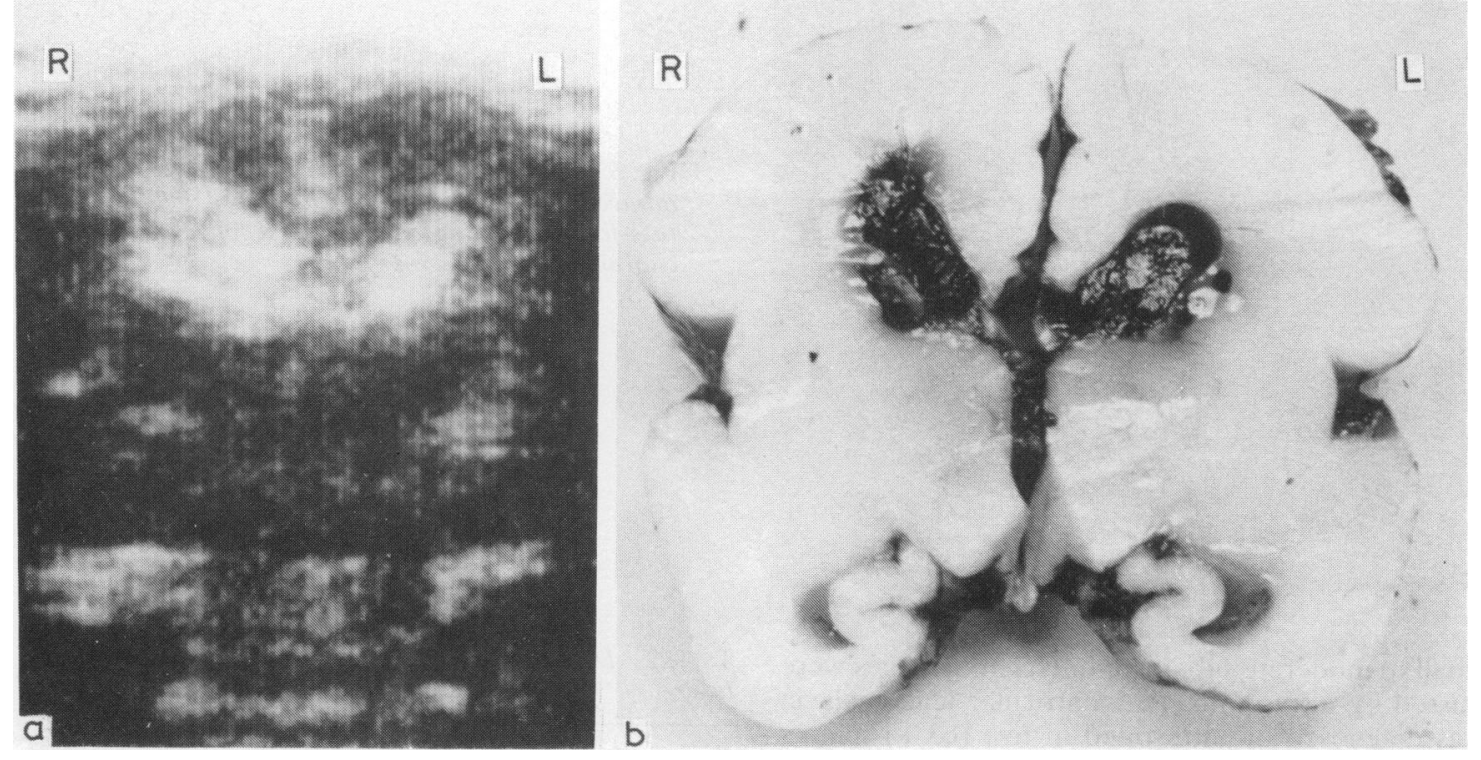

Fig. 2 Bilateral intraventricularand germinal layerhaemorrhages (a)-diagnosed on ultrasound; (b)-confirmed at necropsy in respect of site and size.

pically at necropsy (Fig. 3). The diagnostic accuracy for intracranial haemorrhage was $97 \%$. The two false negative errors occurred because the haemorrhages were out of the 'visual' range of the scanner, at the tip of the frontal lobe in one and at the extreme posterior aspect of the occipital lobe in the other. Five massive subarachnoid haemorrhages were diagnosed by ultrasound but the remaining 11 

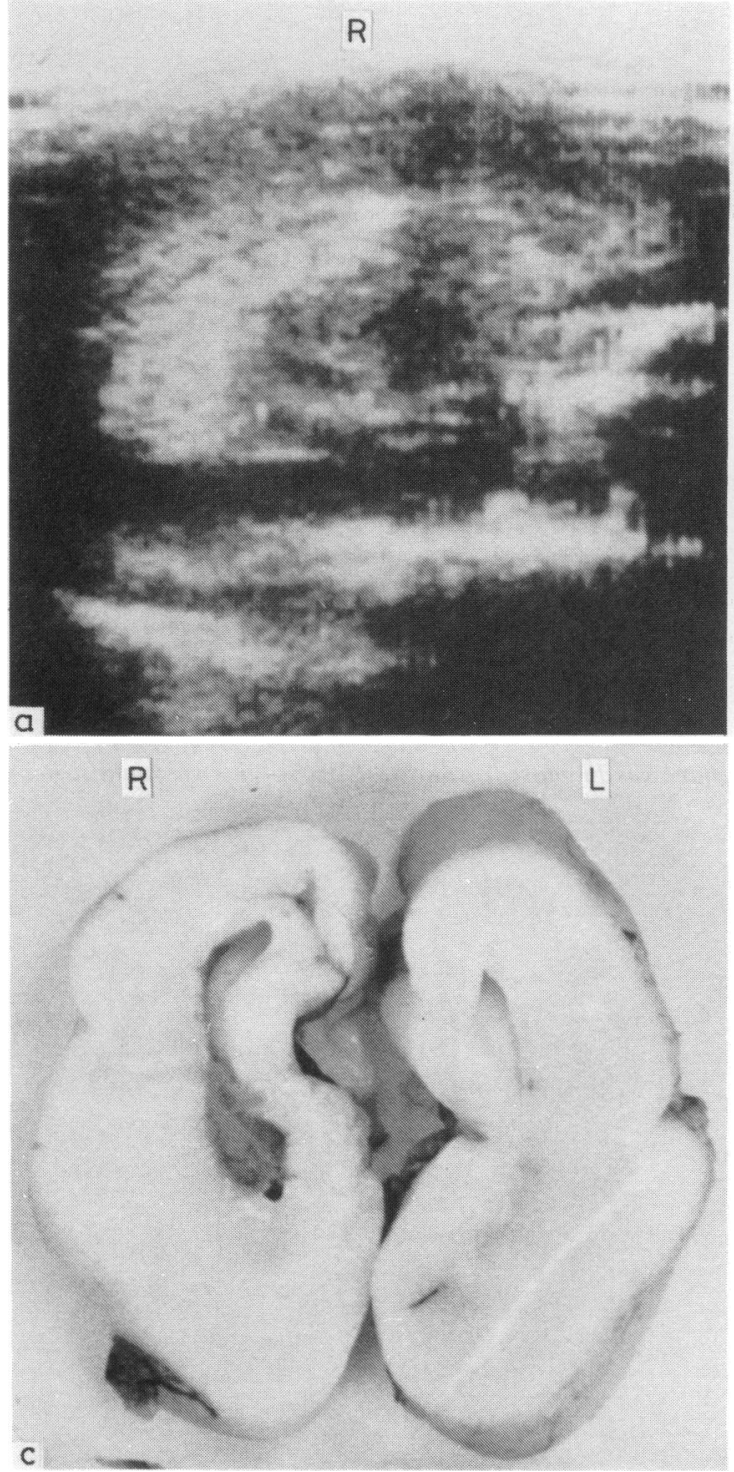

small to moderate subarachnoid haemorrhages were missed by ultrasound. Periventricular leucomalacia was diagnosed by ultrasound in five $(63 \%)$ infants including three with cystic degeneration. False negative errors occurred when periventricular leucomalacia was microscopic or when the cysts occurred out of the range of the scanner, far posteriorly or far anteriorly.

Data from the 77 neonates in the germinal layer study are shown in Table 2 . All but $6(8 \%)$ neonates were of appropriate weight for gestational age. The

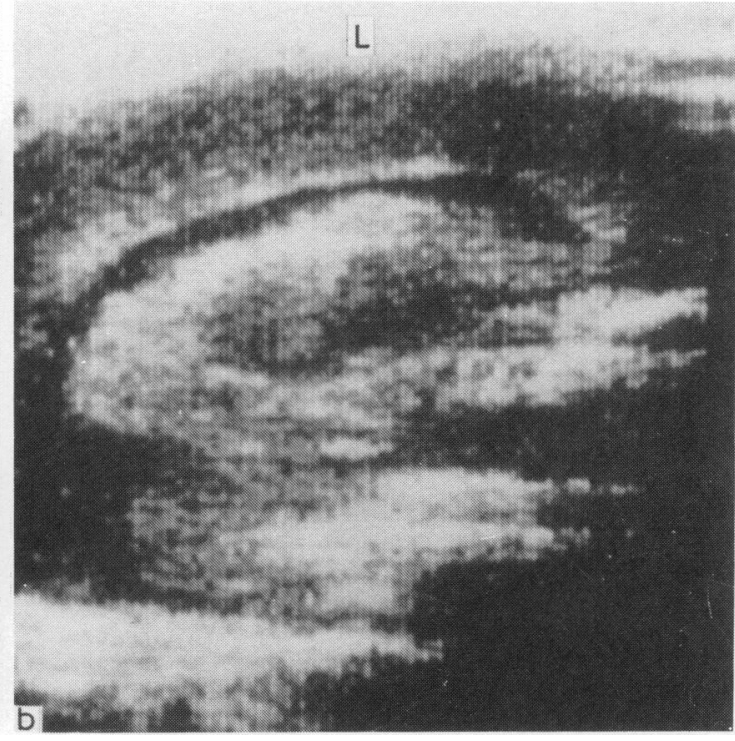

Fig. 3 False positive error: (a) and (b)-right intraventricular haemorrhage diagnosed on ultrasound; (c)-found to be due to pronounced congestion of right choroid plexus at necropsy.

Table 2 Data from 77 neonates with an intact germinal layer

\begin{tabular}{|c|c|c|c|}
\hline$N_{1}$ & $\begin{array}{l}\text { Gestation } \\
\text { (wks) }\end{array}$ & $\begin{array}{l}\text { Birthweight }(g) \\
\text { Me'an }(S D)\end{array}$ & $\begin{array}{l}\text { Width of germinal } \\
\text { laver (mm) } \\
\text { Mean (SD)) }\end{array}$ \\
\hline 14 & $23-24$ & $548(10.3)$ & $2.54(11.56)$ \\
\hline 10 & $25-26$ & $774(149)$ & $2.04(1) \cdot 62)$ \\
\hline 9 & $27-28$ & $9.38(225)$ & $2.17((1.99)$ \\
\hline 11 & $29-30$ & $1219(370)$ & $1.73(0.71)$ \\
\hline 15 & $31-33$ & $1668(522)$ & $1.45(1) .52)$ \\
\hline 10 & $33-34$ & $1932(+24)$ & 1.19 \\
\hline 8 & $35-36$ & $20016(576)$ & $(0.50)(0.26)$ \\
\hline
\end{tabular}


four small for gestational age and the two large for gestational age neonates were not excluded as the measurements of their germinal layer did not alter significantly the mean or standard deviation of any gestational group.

The germinal layer was most prominent in infants of the youngest gestational age ( 23 to 24 weeks); it was half the size at 31 to 32 weeks' gestation and had almost completely involuted at 35 to 36 weeks' gestation.

\section{Discussion}

This study confirmed a high diagnostic accuracy of cerebral ultrasound imaging. Three sources of error were identified. The first error was due to the limitation imposed by the spatial resolution of the ultrasound scanner-microscopic or macroscopic haemorrhages less than $3 \mathrm{~mm}$ in size cannot be visualised. The resolution of sector scanners is likely to be better than the linear array machine we used but correlation with necropsy findings in the diagnosis of periventricular haemorrhage and periventricular leucomalacia has not yet been reported.

The second error was due to vascular congestion of the germinal layer, the choroid plexus or the periventricular area mimicking the appearance of haemorrhage on the ultrasound imaging. Congestion is sometimes seen as pulsating bright echoes. ${ }^{8}$ When this is present it can differentiate congestion from haemorrhage. Congestion, however, may be readily differentiated from haemorrhage by serial scanning, when the 'haemorrhage' seen previously may disappear a day or two later (Fig. 4). Ultrasound findings in the extremely preterm neonate thought to have a small or moderately sized periventricular haemorrhage should be confirmed, therefore, by a repeat scan one or two days later. Serial scanning will also show the typical evolution of haemorrhages-fresh bleeding, clot retraction, autolysis, and resolution-if the infant survives long enough for this characteristic pattern to evolve. ${ }^{9-11}$

The third error was due to haemorrhages or cysts that occurred in areas poorly visualised by or inaccessible to the ultrasound scanner. This error should occur less frequently with the current generation of sector scanners. Real time linear array ultrasound imaging does not detect small subarachnoid haemorrhages or intracerebral haemorrhages beyond the periventricular region.

Progressive involution of the germinal layer with increasing gestational age was paralleled by a reduction in the incidence of periventricular haemorrhage with increasing gestational age found
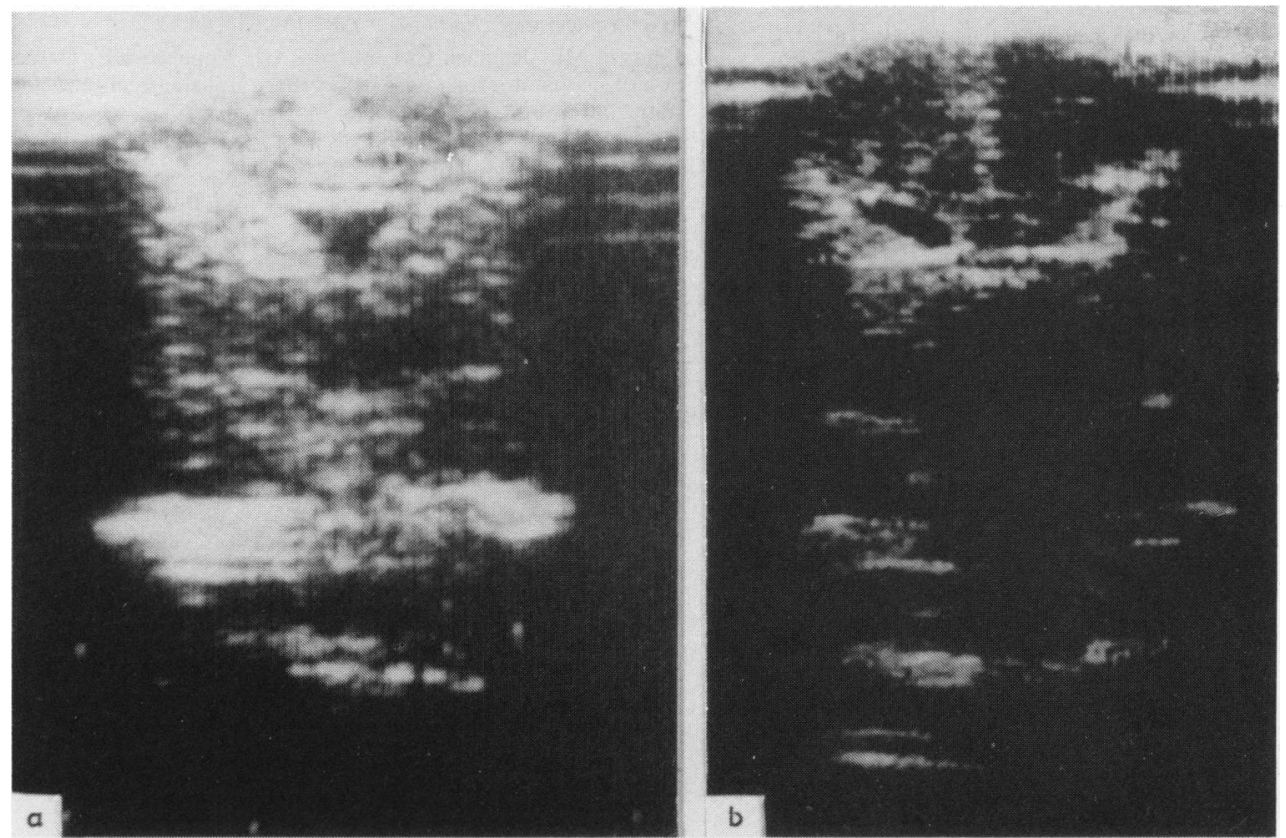

Fig. 4 (a)-congestion of the right germinal layer, mimicking germinal laver haemorrhage, seen on ultrasound on day 1 ; (b) - same infant scanned on day 2, the 'haemorrhage had vanished and did not reappear. 


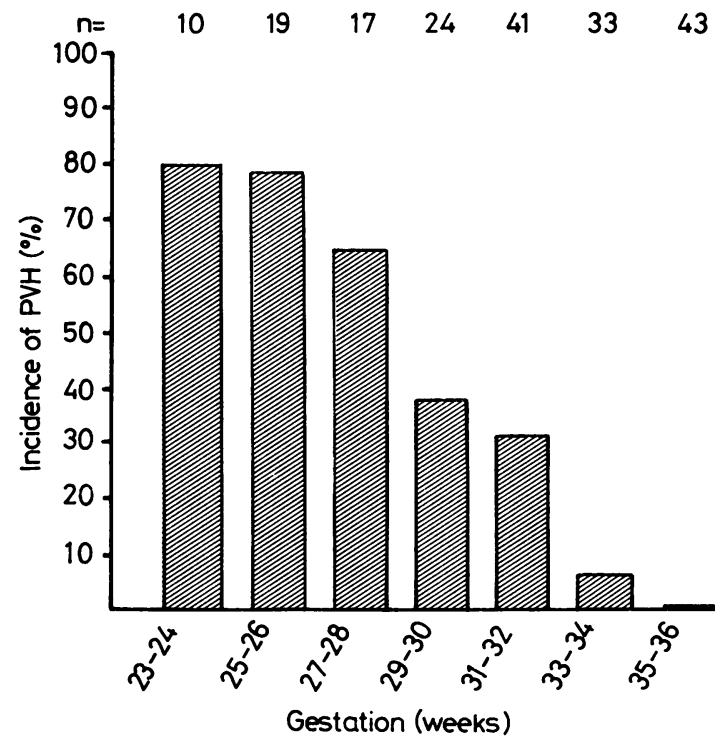

Fig. 5 Incidence of periventricular haemorrhage in 187 infants of gestational age between 23 and 36 weeks.

in a prospective cerebral ultrasound study of 187 preterm infants (Fig. 5). At 23 to 24 weeks' gestation, when the germinal layer was most extensive, the incidence of periventricular haemorrhage was $80 \%$. At 31 to 32 weeks' gestation when the size of the germinal layer had decreased by half. the incidence of periventricular haemorrhage had drupped to $32 \%$. By the time the germinal layer had almost involuted at 35 to 36 weeks' gestation the incidence of periventricular haemorrhage had dropped to zero. The positive correlation of germinal layer involution with the falling incidence of periventricular haemorrhage supports the hypothesis that the germinal layer is necessary for periventricular haemorrhage to develop in the preterm neonate. ${ }^{12}$

\section{References}

' Szymonowicz W, Yu V'YH. Timing and evolution of periventricular hacmorrhage in infants weighing $1250 \mathrm{~g}$ or less at birth. Arch Dis Child 1984:59:7-12.

2 Pape KE. Bennett-Britton S. Szymonowicz W. Martin DJ. Fitz CR. Becker L Diagnostic accuracy of neonatal brain imaging: a postmortem correlation of computer tomography and ultrasound scans. J Pediatr 1983;102:275-80.

${ }^{3}$ Thorburn RJ. Lipscomb AP, Reynolds EOR, et al. Accuracy of imaging of the brains of new-born infants by linear array real-time ultrasound. Early Hum Dev 1982:6:31-46.

+ Babcock DS. Ham BK. The accuracy of high resolution. real-time ultrasonography of the head in infancy. Radiology 1981;139:665-76

5 Thorburn RJ, Lipscomb AP, Stewart AL. Reynolds EOR, Hope PL. Timing and antecedents of periventricular haemorrhage and of cerebral atrophy in very preterm infants. Early Hum Dev 1982;7:221-38.

' Szymonowicz W. Yu VYH. Wilson FE. Antecedents of periventricular haemorrhage in infants weighing $1250 \mathrm{~g}$ or less at birth. Arch Dis Child 1984:59:13-7.

${ }^{7}$ Hambleton G. Wigglesworth JS. Origin of intraventricular hacmorrhage in the preterm infant. Arch Dis Child 1976;51:651-9.

* Levene MI, Wigglesworth JS. Dubowitz V. Congestive periventricular leucomalacia in the neonate: a real-time ultrasound study. Proceedings of second conference on periventricular-intraventricular haemorrhage. 1982:497-512.

"Silverboard G, Horder MH. Ahmann PA. Lazzara A. Schwartz JF. Reliability of ultrasound in diagnosis of intracerebral hemorrhage and posthemorrhagic hydrocephalus: comparison with computed tomography. Pediatrics 1980);66:507-14.

1" Sauerbrei EE, Digney M. Harrison PD. Cooperberg PL. Ultrasonic evaluation of neonatal intracranial hemorrhage and its complications. Radiology 1981;139:667-85.

"Johnson ML. Rumack CM. Mannes EJ. Appareti KE. Detection of neonatal intracranial hemorrhage utilizing real-time and static ultrasound. JCU 1981:9:427-31.

12 Pape KE, Wigglesworth JS. Hemorrhage, ischemia and the perinatal brain. London: Heinemann and Spastics International Medical Publications. 1979. (Clinics in Developmental Medicine Nos 69/70).

Correspondence to Dr V Y H Yu, Qucen Victoria Medical Centre. 172 Lonsdale Strect. Melbourne, Victoria 3(M)0, Australia.

Received 6 March 1984 